J. Appl. Numer. Optim. 1 (2019), No. 3, pp. 217-241

Available online at http://jano.biemdas.com

https://doi.org/10.23952/jano.1.2019.3.03

\title{
VARIATIONAL PRINCIPLES IN SET OPTIMIZATION WITH DOMINATION STRUCTURES AND APPLICATION TO CHANGING JOBS
}

\author{
TRUONG QUANG BAO ${ }^{1, *}$, ANTOINE SOUBEYRAN ${ }^{2}$ \\ ${ }^{1}$ Department of Mathematics and Computer Science, Northern Michigan University, \\ 1401 Presque Isle Avenue, Marquette, MI 49855, USA \\ ${ }^{2}$ Aix-Marseille University (Aix-Marseille School of Economics), CNRS \& EHESS, Marseille 13002, France \\ Dedicated to Professor Christiane Tammer on her 60th birthday.
}

\begin{abstract}
This paper is devoted to new versions of Ekeland's variational principle in set optimization with domination structure, where set optimization is an extension of vector optimization from vector-valued functions to set-valued maps using Kuroiwa's set-less relations to compare one entire image set with another whole image set, and where domination structure is an extension of ordering cone in vector optimization; it assigns each element of the image space to its own domination set. We use Gerstewitz's nonlinear scalarization function to convert a set-valued map into an extended real-valued function and the idea of the proof of Dancs-Hegedüs-Medvegyev's fixed-point theorem. Our setting is applicable to dynamic processes of changing jobs in which the cost function does not satisfy the symmetry axiom of metrics and the class of set-valued maps acting from a quasimetric space into a real linear space. The obtained result is new even in simpler settings.
\end{abstract}

Keywords. Nonlinear scalarization function; Set optimization; Set-less relations; Variational principles; Changing jobs.

2010 Mathematics Subject Classification. 49J53, 65K10, 90C26.

\section{INTRODUCTION}

Set optimization is an extension of vector optimization. Given a set-valued map $F: X \rightrightarrows Y$ from a nonempty set $X$ into a real linear space $Y$ and an ordering cone $C$ of $Y$. There are two main approaches to define minimizers of the set-valued map $F$ with respect to the ordering cone $C$.

The vector-based approach (the best element of the image set) follows directly from the definition of minimizers of vector-valued functions. Precisely, denoting the ordering relation on $Y$ under consideration by " $\leq C$ ", we have its description

$$
y_{1} \leq_{C} y_{2} \text { if and only if } y_{1} \in y_{2}-C .
$$

Then, $\bar{x} \in X$ is called a (vector-based) minimizer of $F$ if there is $\bar{y} \in F(\bar{x})$ such that there is no $y \in F(X) \backslash\{\bar{y}\}$ such that $y \leq_{C} \bar{y}$.

\footnotetext{
${ }^{*}$ Corresponding author.
}

E-mail addresses: btruong@nmu.edu (T.Q. Bao), antoine.soubeyran@gmail.com (A. Soubeyran).

Received August 20, 2019; Accepted November 26, 2019. 
The set-based approach (the best set of all image sets) introduced by Kuroiwa [38] (compare [29] and references therein) via appropriate ordering relations among which is the so-called lower set-less preorder. Denoting this ordering relation by $\leq_{C}^{l}$, we have their descriptions

$$
A \leq_{C}^{l} B \text { if and only if } A+C \supseteq B .
$$

Then, $\bar{x} \in X$ is a (set-based) lower set-less minimizer of $F$ with respect to $C$ if there is no $x \in X$ with $F(x) \neq F(\bar{x})$ such that $F(x) \leq_{C}^{l} F(\bar{x})$.

We use the name set optimization for optimization with set-valued objective maps working with Kuroiwa's set-less relations and the name set-valued optimization when using the usual (Pareto) ordering relation in vector optimization. Recently, there has been an increasing interest in set optimization; see the books [14, 28, 32, 43].

Vector optimization with ordering structure is another extension of vector optimization from a fixed ordering cone to a domination structure; i.e., each element of the image space has its own ordering cone. The solution concepts in vector optimization with variable ordering structures was introduced by $\mathrm{Yu}$ [56] and then was studied in many publications; see, e.g., [12, 13]. This concept is much more general than Pareto efficiency and is crucial in many applications to decision making, games, etc.; see the references above. Recently, set-valued and set optimization with variable ordering structure have been extensively studied in $[5,6,7,8,9,10,18,19,33,34]$ and references therein.

In set-valued optimization with ordering structure, many versions of Ekeland's variational principle have been formulated; for example, $[6,7,10]$ in quasimetric spaces and [4] in pseudo quasimetric spaces with applications in behavioral sciences. There have been several versions of Ekeland's variational principle in set optimization with fixed ordering cone. In particular, in [30], Ha established versions of Ekeland's variational principle for set-valued maps in terms of Kuroiwa's set optimization criterion by using the method of cone extensions. Inspired by Ha's work, Qiu [54] obtained an improvement of Ha's result by relaxing the lower boundedness condition imposed on objective maps. It was further extended in $[35,55]$. However, there has, to the best of our knowledge, no extensions/generalizations to set optimization with ordering structure. Our main objective of this paper is a new version of Ekeland's variational prinicple for set-valued maps in terms of the set-less ordering relation. Our motivation is its application to career development theories; in particular, changing job process. Using the recent variational rationality approach of human dynamics, which focuses the attention on the main concept of worthwhile move [45, 46, 47, 48, 49], we can give an intuitive answer to the initial question: "why changing job can be hard?" which happens as long as motivation to change job is not high enough with respect to resistance to change job. That is, when changing job is not a worthwhile move. Then, allowing a worker to make a succession of worthwhile moves to change from doing a job to doing another job along a succession of periods, we show how the Ekeland variational principle for set-valued mappings with set-less relations gives sufficient conditions for the existence of traps which represent the ends of career development processes.

We use the idea in the proof of extended versions of Dancs-Hegedüs-Medvegyev's fixedpoint theorem in [11]; for more details, see $[6,7,10]$ that were used to establish versions of Ekeland's variational principles in set-valued optimization with ordering structure. In addition, we use marginal functions used in $[30,54]$ associated with Gerstewitz' (nonlinear separation) scalarization function introduced in [21, 22] (see also Krasnoselskii [27] for assertions in the context of operator theory and compare the scalar optimization problem by Pascoletti, Serafini 
[52]) is an important tool in nonconvex vector optimization. The advantage of such a nonlinear scalarization function is a weaker boundedness assumption imposed on set-valued maps in comparison with linear scalarization functions.

The paper is organized as follow. Section 2 is devoted to basic definitions in quasimetric spaces which play the role of cost of change in application, domination structures which can be viewed as preferences of decision makers, and optimization concepts in set optimization with domination structures. We also present a nonlinear scalarization tool and its important properties. Section 3 contains the formulation and the detailed proof of the variational principle discussed above. We also compare it with existing results; it is new even for extended-realvalued functions in quasimetric spaces. Section 4 is devoted to a major application of changing jobs.

\section{BASIC DEFINITIONS AND PRELIMINARIES}

Since we intend to apply our variational principles to worthwhile stay and change approach which might be viewed as a general model of human behavior, the domain space needs to be a quasi-metric space; see the last section for justifications of the chosen spaces. We follow notations in [4]; see also [6, 11].

Definition 2.1. (quasimetrics). A QUASIMETRIC on a nonempty set $X$ is a bifunction $q: X \times$ $X \rightarrow \mathbb{R}$ satisfying all axioms for a metric with the possible exception of symmetry. Precisely, for every $x, u, w \in X$ one has

(q1): $q(x, u) \geq 0 \quad$ (positivity);

(q2): $q(x, u)=0 \Longleftrightarrow x=u \quad$ (identity of indiscernibles);

(q3): $q(x, u) \leq q(x, w)+q(w, u) \quad$ (subadditivity, or triangle inequality).

In contrast to metric spaces, there are several notions of convergence in (bitopological) quasimetric spaces; see $[4,31]$ and the references therein. For the sake of brevity, we present concepts used in the subsequent sections.

Definition 2.2. (notations in pseudo-quasimetric spaces). Let $\left\{x_{n}\right\}$ be a sequence in a quasimetric space $(X, q)$. We say that:

(i) $\left\{x_{n}\right\}$ is FORWARD-CONVERGENT if there exists $x_{*} \in X$ such that $q\left(x_{n}, x_{*}\right) \rightarrow 0$. Such an element $x_{*}$ is called a forward-limit. We denote $\overrightarrow{\left\{x_{n}\right\}}$ as the collection of forward-limits of the sequence $\left\{x_{n}\right\}$;

(ii) $\left\{x_{n}\right\}$ is FORWARD-CAUCHY if for each $\varepsilon>0$ there is an integer $N_{\varepsilon} \in \mathbb{N}$ such that $q\left(x_{n}, x_{n+j}\right)<\varepsilon$ for all $n \geq N_{\varepsilon}$ and $j \in \mathbb{N}$;

(iii) the space is FORWARD-COMPLETE if every forward-Cauchy sequence is forward-convergent to some forward-limit;

(iv) the space is forward-Hausdorff if every forward-convergent sequence has the unique forward-limit. 
It is important to emphasize that a forward-convergent sequence in a quasimetric space is not unique. Consider the quasimetric space $([0,1], q)$, where $q$ is defined by

$$
q(x, u)= \begin{cases}x-u & \text { if } x \geq u \\ 1+x-u & \text { if } x<u \text { but }(x, u) \neq(0,1) \\ 1 & \text { if }(x, u)=(0,1)\end{cases}
$$

Then, the sequence $\left\{x_{n}\right\}$ with $x_{n}=1 / n$ has two forward-limits 0 and 1 .

Definition 2.3. (generalized-Picard sequences, [16]). Let $X$ be a nonempty set, $S: X \rightrightarrows X$ a set-valued mapping, and $\varphi: X \rightarrow \mathbb{R} \cup\{ \pm \infty\}$ a function. We say that a sequence $\left\{x_{n}\right\}$ in $X$ is a GENERALIZED PICARD SEQUENCE of $S$, if $x_{n+1} \in S\left(x_{n}\right)$ for all $n \in \mathbb{N}$.

Definition 2.4. (strict-decreasingly forward-lower-semicontinuous functions, [4, Definition 9]). Let $(X, q)$ be a quasimetric space and $\varphi: X \rightarrow \mathbb{R} \cup\{ \pm \infty\}$ be an extended real-valued function on $X$. The function $\varphi$ is said to be STRICT-DECREASINGLY FORWARD-LOWERSEMICONTINUOUS, if for every forward-Cauchy sequence $\left\{x_{n}\right\}$ such that $\left\{\varphi\left(x_{n}\right)\right\}$ is strictly decreasing, one has

$$
\forall y_{*} \in \overrightarrow{\left\{x_{n}\right\}}, \varphi\left(y_{*}\right) \leq \lim _{n \rightarrow \infty} \varphi\left(x_{n}\right),
$$

where $\overrightarrow{\left\{x_{n}\right\}}$ is the collection of forward-limits of the sequence $\left\{x_{n}\right\}$.

Let us present [4, Corollary 3] in the quasi-metric setting; it was formulated in pseudoquasimetric spaces which allowing the quasidistance between two distinct element zero and its assumptions were imposed on the set-valued map $S$ introduced below.

Lemma 2.1. (a forward version of Ekeland's variational principle, cf. [4, Corollary 3]) Let $(X, q)$ be a quasimetric space and $\varphi: X \rightarrow \mathbb{R} \cup\{+\infty\}$ be a function and $x_{0} \in \operatorname{dom}(\varphi)$. Consider a set-valued map $S: X \rightrightarrows X$ with images

$$
S(x):=\{u \in X: \varphi(u)+q(x, u) \leq \varphi(x)\} .
$$

Assume that

(A1) $\varphi$ is bounded from below on $S\left(x_{0}\right)$;

(A2) $\varphi$ is strict-decreasingly forward-lower-semicontinuous on $S\left(x_{0}\right)$ in the sense of Definition 2.4;

(A3) Every forward-Cauchy sequence $\left\{x_{n}\right\}$ such that $\left\{\varphi\left(x_{n}\right)\right\}$ is strictly decreasing is forwardconvergent; it is automatic in forward-complete spaces.

Then, there exists $x_{*} \in X$ satisfying

(i) $\varphi\left(x_{*}\right)+q\left(x_{0}, x_{*}\right) \leq \varphi\left(x_{0}\right)$;

(ii) $\forall x \in X \backslash\left\{x_{*}\right\}, \varphi(x)+\lambda q\left(x_{*}, x\right)>\varphi\left(x_{*}\right)$.

The differences between this version and the original principle in metric spaces are: (1) the metric space enjoys condition (A3); it is not necessarily complete; and (2) the function $\varphi$ enjoys the strict-decreasing lower-semicontinuity property which is weaker than lower-semicontinuity one; see [4, Example 7] for examples.

Next, we present basic concepts and notations in set optimization with domination structure, which is a far-reaching form of vector optimization in three directions: 
(1) We use domination sets instead of ordering cones in order to define the known preorder in vector optimization.

(2) Domination sets vary from decisions of decision makers.

(3) We use the so-called lower set-less preorder (in order to compare one set with another sets) to define minimizers of set-valued objective maps.

Definition 2.5. Let $X$ be a nonempty set, $Y$ be a real linear space, and $F: X \rightrightarrows Y$ be a set-valued map.

(i) A nonempty set $D$ of $Y$ is called a domination set in $Y$ if $0 \in D$ and $D+D \subseteq D$.

(ii) A set-valued map $\mathscr{D}: X \rightrightarrows Y$ is called a domination structure in $Y$ if for every $x \in X$, $\mathscr{D}(x)$ is a domination set.

In [18], the authors studied convex-cone-valued domination structures. In a majority of publications in the literature, $X=Y$ and domination sets are convex cones; see, for example, $[6,9,10,19,56]$ and the references therein.

Definition 2.6. (binary relations in set optimization with domination structure). Let $F$ : $X \rightrightarrows Y$ be a set-valued map and $\mathscr{D}$ be a domination structure and $x, u \in \operatorname{dom} F$.

(i) We say that $F(u)$ is said to be pre-lower-set-less than $F(x)$ or $F(u)$ is less than $F(x)$ with respect to the pre-lower-set-less binary relation $\leq_{\mathscr{D}}^{l, e}$ and write $F(u) \leq_{\mathscr{D}}^{l, e} F(x)$ if $F(u)+\mathscr{D}(x) \supseteq F(x)$.

(ii) We say that $F(u)$ is said to be post-lower-set-less than $F(x)$ and write $F(u) \leq_{\mathscr{D}}^{l, n} F(x)$ if $F(u)+\mathscr{D}(u) \supseteq F(x)$.

When $\mathscr{D}$ is a constant domination structure; i.e., $\mathscr{D}(x)=D$ for all $x \in X$ for some domination set $D$ in $Y$, both pre- and post-lower-set-less binary relations are identical. Assume, in addition, that $D$ is a cone, they reduces to the known lower-set-less preorder introduced by Kuroiwa in [38]. It is important to note that we could study other binary relations in set optimization with domination structure corresponding to other relations in set optimization with ordering cone/constant domination structure in [29, 38].

When $F=f: X \rightarrow Y$ is a single-valued function, they reduce to the so-called efficient and domination binary relations with respect to the domination structure $\mathscr{P}: Y \rightrightarrows Y$ such that $\mathscr{D}=$ $\mathscr{P} \circ f$ in vector optimization with binary structure. See, for example, $[8,56]$.

When $\mathscr{D}$ is a constant domination structure and $F=f: X \rightarrow Y$ is a single-valued function, they agree with the binary relation $\leq_{D}$ in $Y$ which is defined by

$$
v \leq_{D} y: \Longleftrightarrow v \in y-D
$$

Assume in addition that $D=C$ is a convex cone, then $\leq_{C}$ is a preorder in $Y$. Assume, in addition, that $C$ is pointed, then $\leq_{C}$ is a partial order in $Y$. It is standard in vector optimization; known also as multiobjective optimization theory, and various applications; see the books [23, 28, 41, 42]. Note that two requirements of domination sets guarantee the reflexivity and transitivity properties of the binary relation $\leq_{D}$; in the other words, $\leq_{D}$ is a preorder. Such a domination set is $D=\{0\} \cup\left\{d \in \mathbb{R}_{+}^{2}: d_{1}+d_{2} \geq 1\right\}$, which is not a cone.

The next proposition provides a sufficient condition guaranteeing that $\leq_{\mathscr{D}}^{l, e}$ is a preorder. 
Proposition 2.1. The pre-lower-set-less binary relation introduced in Definition 2.6 is a preorder in the collection of sets

$$
\{F(x): x \in W\} \text { with } W \subseteq \operatorname{dom} F
$$

if the transitivity condition

$$
\forall u, v \in W, F(u) \leq_{\mathscr{D}}^{l, e} F(x) \Longrightarrow \mathscr{D}(u)+\mathscr{D}(x) \subseteq \mathscr{D}(x)
$$

holds.

Proof. Obviously, the reflexivity property of $\leq_{\mathscr{D}}^{l, e}$ holds due to the definition of $\mathscr{D}$. To check the transitivity property, fix arbitrary three set $F(x), F(u)$ and $F(w)$ satisfying $F(u) \leq_{\mathscr{D}}^{l, e} F(x)$ and $F(w) \leq_{\mathscr{D}}^{l, e} F(u)$ and thus

$$
F(u)+\mathscr{D}(x) \supseteq F(x) \text { and } F(w)+\mathscr{D}(u) \supseteq F(u) .
$$

Combining these two inclusions together while taking into account the transitivity condition in (2.2) $\mathscr{D}(u)+\mathscr{D}(x) \subseteq \mathscr{D}(x)$, we have

$$
F(w)+\mathscr{D}(x) \supseteq F(w)+\mathscr{D}(u)+\mathscr{D}(x) \supseteq F(x) .
$$

Therefore, we have $F(w) \leq_{\mathscr{D}}^{l, e} F(x)$. The proof is complete.

Although we study new versions of Ekeland's variational principle for set-valued maps with respect to the pre-lower-set-less preorder only, the counterpart of the obtained results with respect to the post-lower-set-less preorder could be formulated in a similar way.

Definition 2.7. (minimality concepts in set optimization). Let $F: X \rightrightarrows Y$ be a set-valued map, $\mathscr{D}$ be a domination structure and $\bar{x} \in \operatorname{dom} F$. We say that

(i) $\bar{x}$ is a pre-lower-set-less minimizer of $F$ with respect to the domination structure $\mathscr{D}$; in short, $\leq_{\mathscr{D}}^{l, e}$-minimizer, if for every $x \in \operatorname{dom} F, F(x) \leq_{\mathscr{D}}^{l, e} F(\bar{x})$ implies $F(\bar{x}) \leq_{\mathscr{D}}^{l, e} F(x)$.

(ii) $\bar{x}$ is a strict $\leq_{\mathscr{D}}^{l, e}$-minimizer, if for every $x \in \operatorname{dom} F, F(x) \leq_{\mathscr{D}}^{l, e} F(\bar{x})$ implies $x=\bar{x}$.

(iv) Similarly, we could define the counterparts of (respectively, strict) $\leq_{\mathscr{D}}^{l, n}$-minimizers.

We will end this section with an important nonlinear scalarization tool from [22] by Gerstewitz (Tammer). Let $Y$ be a real linear space and $A$ be a nonempty set in $Y$. The set $A$ is said to be pointed if $A \cap(-A) \subseteq\{0\}$, and a cone if $\lambda a \in A$ for all $a \in A$ and $\lambda \geq 0$. A cone $A$ is called nontrivial if $A \neq\{0\}$ and $A \neq Y$. A nonempty set $A \subseteq Y$ is said to be free-disposal with respect to a convex cone $C$ if $A+C=A$. This notion was introduced by Debreu in the setting of mathematical economics.

Given a nonempty set $A$ in $Y$, the notations core ${ }_{k} A, \operatorname{vcl}_{k} A$ stands for the algebraic interior and the vector closure of $A$ in the direction $k$

$$
\begin{gathered}
\operatorname{vcl}_{k} A:=\{y \in Y: \forall \tau>0, \exists t \in[0, \tau], y+t k \in A\}, \\
\operatorname{core}_{k} A:=\{y \in Y: \forall \tau>0, \exists t \in[-\tau, \tau], y+t k \in A\} .
\end{gathered}
$$

For more results on directionally vector closedness and relationships with vector closedness and topological closedness, see [25] and the references therein.

Lemma 2.2. ([25, Lemma 2.3]). Assume that A is free-disposal with respect to cone $(k)$. Then, we have

$$
\operatorname{core}_{k} A=\operatorname{vcl}_{k} A+(0,+\infty) k=A+(0,+\infty) k
$$


Definition 2.8. Let $Y$ be a real linear space, $k \in Y \backslash\{0\}$, and $D \subseteq Y$ be a nonempty set being freedisposal with respect to cone $(k)$. The Gerstewitz scalarization function $\varphi_{k, A}: Y \rightarrow \mathbb{R} \cup\{ \pm \infty\}$ associated with $k$ and $A$ is defined by

$$
\forall y \in Y, \varphi_{k, A}(y):=\inf \{t \in \mathbb{R}: y \in t k-A\} .
$$

It is important to mention that the Gerstewitz scalarization function was originally defined in real topological spaces for (topologically) closed sets being free-disposal w.r.t. the scalarization direction. In [25], it was extended to real linear spaces for any nonzero direction and any nonempty set. We could find a function of the original form which gives the same outputs.

In vector optimization dealing with minimization, one needs to ensure that the values of a function is not equal to $-\infty$. It is not difficult to check from its definition, $\varphi_{k, A}(y)=-\infty$ if and only if $-y+\mathbb{R} k \subseteq A$. The left hand side is the line going through $-y$ and parallel to the direction $k$ and thus $\varphi_{k, D}$ is proper if and only if $A$ does not contain lines parallel to $k$. For the main properties of Gerstewitz's scalarization functions, see [23, 25].

Lemma 2.3. ([25, Lemma 2.2]). A scalarization function $\varphi_{k, A}$ has the following properties:

(a) $\varphi_{k, A}(y)<+\infty$ if and only if $y \in \mathbb{R} k-A$ and $\varphi_{k, A}(y)>-\infty$ if and only if $-y+\mathbb{R} k \subseteq A$.

(b) $\forall y \in Y, \forall t \in \mathbb{R}, \varphi_{k, A}(y+t k)=\varphi_{k, A}(y)+t$.

(c) $\operatorname{lev}\left(\varphi_{k, A},<, 0\right)=(-\infty ; 0) k-\operatorname{vcl}_{q} A$.

(d) $\operatorname{lev}\left(\varphi_{k, A}, \leq, 0\right)=-$ cone $(k)-\operatorname{vcl}_{k} H=-\operatorname{vcl}_{k} H$.

(e) $\varphi_{k, A}$ is nondecreasing with respect to $\leq_{\Theta}$ if and only if $A+\Theta \subseteq A$.

Given a set-valued mapping $F: X \rightrightarrows Y$. The marginal function $m: X \rightarrow \mathbb{R} \cup\{ \pm \infty\}$ of the set-valued map $F$ via the scalarization function $\varphi_{k, A}$ is defined by

$$
m(x):=\inf \left\{\varphi_{k, A}(y) \mid y \in F(x)\right\} .
$$

\section{EKELAND'S VARIATIONAL PRINCIPLE}

This section is devoted to new versions of Ekeland's variational principle for set-valued map in set optimization with domination structures in the following setting. Let us state the standing assumptions in this section:

(H1) $(X, d)$ is a quasimetric. $Y$ is a real linear space. $k$ is a nonzero element in $Y . F: X \rightrightarrows Y$ is a set-valued map. $\mathscr{D}: X \rightrightarrows Y$ is a domination structure and $\leq_{\mathscr{D}}^{l, e}$ is the pre-lower-setless binary relation introduced in Definition 2.6. Consider the perturbed set-valued map $F_{X}: X \rightrightarrows Y$ with images

$$
\forall u \in X, F_{x}(u):=F(u)+q(x, u) k \text { with } F_{x}(x)=F(x) .
$$

Consider the level-set map $W: X \rightrightarrows X$ which is defined via level sets of perturbed maps with respect to $\leq_{\mathscr{D}}^{l, e}$ by

$$
\begin{aligned}
W(x) & :=\operatorname{Lev}\left(F, \leq_{\mathscr{D}}^{l, e}, F_{x}(x)\right)=\left\{u \in X: F_{x}(u) \leq_{\mathscr{D}}^{l, e} F_{x}(x)\right\} \\
& =\{u \in X: F(u)+q(x, u) k+\mathscr{D}(x) \supseteq F(x)\} .
\end{aligned}
$$

Denote $W_{0}=W\left(x_{0}\right)$.

(H2) $\mathscr{D}$ is free-disposal and $k$-vectorially closed on $W_{0}$ with respect to the cone cone $(k)$; i.e.,

$$
\forall x \in W_{0}, \mathscr{D}(x)+\text { cone }(k) \subseteq \mathscr{D}(x) \text { and } \operatorname{vcl}_{k} \mathscr{D}(x)=\mathscr{D}(x) .
$$


$\mathscr{D}$ enjoys the transitivity property

$$
\forall x, u \in W_{0}, F(u) \leq_{\mathscr{D}}^{l, e} F(x) \Longrightarrow \mathscr{D}(u)+\mathscr{D}(x) \subseteq \mathscr{D}(x) .
$$

(H3) $\varphi_{k, A}: Y \rightarrow \mathbb{R} \cup\{ \pm \infty\}$ is a scalarization function with uniform level sets defined in (2.3), where $A \subseteq \mathscr{D}\left(x_{0}\right)$ is a nonempty set being free-disposal with respect to the cone cone $(k)$. The marginal function introduced in (2.4) is finite at $x_{0}$.

(H4) Every forward-Cauchy sequence $\left\{x_{n}\right\}$ such that $\left\{F\left(x_{n}\right)\right\}$ is $<_{\mathscr{D}}^{l, e}$-decreasing is forwardconvergent; this is automatic provided that $(X, q)$ is forward-complete.

Note that we could take $A=\mathscr{D}\left(x_{0}\right)$ as the smallest set. However, we might choose a larger set for $A$ as a solid and convex cone and $k$ is in the interior of $A$, then the the domain of $\varphi_{k, A}$ is the entire space.

In order to capture the variational principle in Lemma 2.1, we need the weak counterpart of the preorder $\leq_{\mathscr{D}}^{l, e}$, which is defined by replacing a domination set by its core with respect to the direction $k$. Precisely, one has

$$
F(u)<_{\mathscr{D}}^{l, e} F(x): \Longleftrightarrow F(u)+\operatorname{core}_{k} \mathscr{D}(x) \supseteq F(x) .
$$

Note that Lemma 2.2 gives core $_{k} \mathscr{D}(x):=\mathscr{D}(x)+(0,+\infty) k$.

Definition 3.1. Let the standing assumptions (H1)-(H3) hold. The set-valued map $F: X \rightrightarrows Y$ is said to be $<_{\mathscr{D}}^{l, e}$-decreasingly forward-lower-semicontinuous if for every forward-convergent sequence $\left\{x_{n}\right\}$ such that the image sequence $\left\{F\left(x_{n}\right)\right\} \subseteq 2^{Y}$ is decreasing with respect to the weak relation $<_{\mathscr{D}}^{l, e}$, there exists a forward-limit $x_{*}$ of the sequence $\left\{x_{n}\right\}$ such that

$$
\forall n \in \mathbb{N}, F\left(x_{*}\right) \leq_{\mathscr{D}}^{l, e} F\left(x_{n}\right) .
$$

It is important to emphasize that the validity of (3.4) for one a forward-limit is sufficient to establish variational principles of Ekeland-type. As a consequence, it improves the strict decreasingly forward-lower-semicontinuity assumption in the Ekeland variational principle in quasimetric spaces.

The function $\varphi(x)=x$ is not strict-decreasingly forward-lower-semicontinuous in the quasimetric space $([0,1], q)$ where $q$ is defined by

$$
q(x, u)= \begin{cases}x-u & \text { if } x \geq u \\ 1+x-u & \text { if } x<u \text { but }(x, u) \neq(0,1) \\ 1 & \text { if }(x, u)=(0,1)\end{cases}
$$

In this space, the strict- $\varphi$-decreasingly forward-Cauchy sequence $\left\{x_{n}\right\}$ with $x_{n}=\frac{1}{n}$ has two forward-limits $x_{*}=0$ and $u_{*}=1$. Obviously,

$$
\liminf _{n \rightarrow \infty} \varphi\left(x_{n}\right)=\lim _{n \rightarrow \infty} \frac{1}{n}=0=\varphi(0)=\varphi\left(x_{*}\right) \neq \varphi(1)=\varphi\left(u_{*}\right) .
$$

This proves that $\varphi$ is not strict-decreasingly forward-lower-semicontinuous in $(X, q)$ in the sense of Definition 2.4, but it is <-deceasingly forward-lower-semicontinuous in $(X, q)$ in the sense of Definition 3.1.

When $(X, q)$ is a metric, the forward-limit set $\overrightarrow{\left\{x_{n}\right\}}$ is singleton and thus it extends the socalled strictly decreasing forward-lower-semicontinuity for the class of functions $\varphi: X \rightarrow \mathbb{R} \cup$ $\{ \pm \infty\}$ in [4, Definition 3.18]; known also as lower-semicontinuity from above in [36]. It also 
extend the concept of decreasing forward-lower-semicontinuity studied in $[6,10]$ for vectorvalued functions.

Before stating our main variational principle, we prove several auxiliary results.

Proposition 3.1. Let the standing assumptions (H1)-(H3) hold. The set-valued map $W$ defined in (3.2) enjoys the following properties:

(W1) $\forall x \in W_{0}, x \in W(x)$.

(W2) $\forall x, u \in W_{0}, u \in W(x) \Longrightarrow F(u) \leq_{\mathscr{D}}^{l, e} F(x)$ and $\mathscr{D}(u)+\mathscr{D}(x) \subseteq \mathscr{D}(x)$.

Assume in addition that $u \neq x$. Then, $F(u)<_{\mathscr{D}}^{l, e} F(x)$.

(W3) $\forall x, u \in W_{0}, u \in W(x) \Longrightarrow W(u) \subseteq W(x)$.

Proof. The conclusion in (W1) is straightforward from the definition of $W$ and $0 \in \mathscr{D}(x)$.

To prove (W2), we fix two arbitrary elements $\forall x, u \in W_{0}$ with $u \in W(x)$. We have

$$
\begin{aligned}
& u \in W(x) \quad \Longleftrightarrow \quad F_{x}(u) \leq{ }_{\mathscr{D}}^{l, e} F_{x}(x) \\
& \Longleftrightarrow \quad F(u)+q(x, u) k+\mathscr{D}(x) \supseteq F(x) \\
& \text { free-disposal } F(u)+\mathscr{D}(x) \supseteq F(x) \text { since } q(x, u) k+\mathscr{D}(x) \subseteq \mathscr{D}(x)
\end{aligned}
$$

clearly verifying $F(u) \leq_{\mathscr{D}}^{l, e} F(x)$. By the transitivity condition $(2.2)$, we have $\mathscr{D}(u)+\mathscr{D}(x) \subseteq$ $\mathscr{D}(x)$.

Assume in addition that $u \neq x$. Then, we have $q(x, u)>0$ and thus

$$
q(x, u) k+\mathscr{D}(x) \subseteq(0,+\infty) k+\mathscr{D}(x)=\operatorname{core}_{k} \mathscr{D}(x) .
$$

Instead of using the free disposal property as above, we use this inclusion to get $F(u)+$ core $_{k} \mathscr{D}(x) \supseteq F(x)$, i.e., $F(u)<_{\mathscr{D}}^{l, e} F(x)$.

To prove (W3), we fix arbitrary elements $x, u, w \in W_{0}$ with $u \in W(x)$ and $w \in W(u)$. By the definition of $W$ and (W2), we have

$$
\begin{array}{r}
F(w)+q(u, w) k+\mathscr{D}(u) \supseteq F(u) \text { and } F(u)+q(x, u) k+\mathscr{D}(x) \supseteq F(x) \\
D(w)+\mathscr{D}(u) \subseteq \mathscr{D}(u) \text { and } D(u)+\mathscr{D}(x) \subseteq \mathscr{D}(x) .
\end{array}
$$

Combining these inclusion, we get

$$
F(w)+q(x, u) k+q(u, w) k+\mathscr{D}(x) \supseteq F(x),
$$

Taking into account the free-disposal property $\mathscr{D}(x)+$ cone $(k) \subseteq \mathscr{D}(x)$ and the subadditivity property of the quasimetric $q(x, u)+q(u, w) \geq q(x, w)$, we arrive at

$$
F(w)+q(x, w) k+\mathscr{D}(x) \supseteq F(x),
$$

clearly verifying that $F_{x}(w) \leq_{\mathscr{D}}^{l, e} F_{x}(x)$ and thus $w \in W(x)$. Since $w$ was arbitrary in $W(u)$, we have $W(u) \subseteq W(x)$.

Proposition 3.2. Let the standing assumptions (H1)-(H3) hold and let $\left\{x_{n}\right\}_{n=0}^{\infty}$ be a generalized Picard sequence of $W$ satisfying

$$
\forall n \in \mathbb{N} \cup\{0\}, q\left(x_{n}, x_{n+1}\right)>0 \text { and } \sum_{n=0}^{+\infty} q\left(x_{n}, x_{n+1}\right)<+\infty \text {. }
$$


Assume that $F$ is $<_{\mathscr{D}}^{l, e}$-decreasingly forward-lower-semicontinuous on $W_{0}$. Then, there is a forward-limit $x_{*}$ of $\left\{x_{n}\right\}$ such that

$$
x_{*} \subseteq \bigcap_{n=0}^{\infty} W\left(x_{n}\right)
$$

where $W$ is defined in 3.2.

Proof. Since $\left\{x_{n}\right\}_{n=0}^{\infty}$ is a a generalized Picard sequence of $W$ with $q\left(x_{n}, x_{n+1}\right)>0$, Proposition 3.1 (W2) proves that $\left\{F\left(x_{n}\right)\right\}_{n=0}^{\infty}$ is $<_{\mathscr{D}}^{l, e}$-decreasing. Since $\sum_{n=0}^{+\infty} q\left(x_{n}, x_{n+1}\right)<+\infty$, the sequence $\left\{x_{n}\right\}$ is forward-Cauchy (see, [4, Proposition 3]) and thus it is forward-convergent due to (H4).

Since $F$ is $<_{\mathscr{D}}^{l, e}$-decreasingly forward-lower-semicontinuous on $W_{0}$, there exists a forward limit $x_{*}$ of the sequence $\left\{x_{n}\right\}$ such that condition (3.4) holds, i.e.,

$$
\forall n \in \mathbb{N} \cup\{0\}, F\left(x_{*}\right)+\mathscr{D}\left(x_{n}\right) \supseteq F\left(x_{n}\right) .
$$

Since $\left\{x_{n}\right\}_{n=0}^{\infty}$ is a generalized Picard sequence of $W$, we have

$$
F\left(x_{n+1}\right)+q\left(x_{n}, x_{n+1}\right) k+\mathscr{D}\left(x_{n}\right) \supseteq F\left(x_{n}\right) .
$$

By Proposition 3.1 (W2), we also have

$$
\forall n \in \mathbb{N} \cup\{0\}, \mathscr{D}\left(x_{n+1}\right)+\mathscr{D}\left(x_{n}\right) \subseteq \mathscr{D}\left(x_{n}\right) .
$$

The subadditivity property of the quasimetric $q$ and the free disposal property of $\mathscr{D}\left(x_{n}\right)$ ensure that

$$
\left(\sum_{i=n}^{n+j-1} q\left(x_{i}, x_{i+1}\right)-q\left(x_{n}, x_{n+j}\right)\right) k+\mathscr{D}\left(x_{n}\right) \subseteq \mathscr{D}\left(x_{n}\right) .
$$

Combining the inclusion in (3.7) for $n-1$ to $n+j$ while taking into account (3.8) and (3.9), we have

$$
F\left(x_{n+j}\right)+q\left(x_{n}, x_{n+j}\right) k+\mathscr{D}\left(x_{n}\right) \supseteq F\left(x_{n}\right) .
$$

This and (3.6) yield

$$
F\left(x_{*}\right)+q\left(x_{n}, x_{n+j}\right) k+\mathscr{D}\left(x_{n}\right) \supseteq F\left(x_{n}\right)
$$

and thus

$$
F\left(x_{*}\right)+q\left(x_{n}, x_{*}\right) k-q\left(x_{n+j}, x_{*}\right) k+\mathscr{D}\left(x_{n}\right) \supseteq F\left(x_{n}\right) .
$$

due to the triangle inequality of the quasimetric $q$ and the free-disposal property of $\mathscr{D}\left(x_{n}\right)$. Since $j$ was arbitrary and $\mathscr{D}\left(x_{n}\right)$ is $k$-vectorially closed, we obtain by passing to limit as $j \rightarrow \infty$

$$
F\left(x_{*}\right)+q\left(x_{n}, x_{*}\right) k+D\left(x_{n}\right) \supseteq F\left(x_{n}\right)
$$

clearly verifying that $x_{*} \in W\left(x_{n}\right)$. Since $n$ was arbitrary, the proof is complete.

Proposition 3.3. Consider the marginal function $m$ and the set-valued mapping $W$ introduced in (3.2) and (2.4), respectively. The following hold:

(i) $\forall x \in W_{0}, m(x)=\inf \left\{\varphi_{k, A}(u): u \in F(x)+\mathscr{D}\left(x_{0}\right)\right\}$.

(ii) If $u \in W(x)$, then $m(u)+q(x, u) \leq m(x)$. Assume in addition that $u \neq x$, then $m(u)<$ $m(x)$.

(iii) If $F(u)+t k+\mathscr{D}\left(x_{0}\right) \supseteq F(x)+\mathscr{D}\left(x_{0}\right)$ for some elements $x, u \in W_{0}$ and a real number $t \in \mathbb{R}$, then we have $m(u)+t \leq m(x)$. 
Proof. To prove (i), take an arbitrary element $x \in W_{0}$. Note that we always have $x \in W_{0}=W\left(x_{0}\right)$ and $\mathscr{D}(x)+\mathscr{D}\left(x_{0}\right) \subseteq \mathscr{D}\left(x_{0}\right)$ and $\mathscr{D}\left(x_{0}\right)+\mathscr{D}\left(x_{0}\right) \subseteq \mathscr{D}\left(x_{0}\right)$. Fix arbitrary elements $y \in F(x)$ and $d \in \mathscr{D}\left(x_{0}\right)$. We have $y \leq \mathscr{D}\left(x_{0}\right) y+d-\mathscr{D}\left(x_{0}\right)$. Since $A+\mathscr{D}\left(x_{0}\right) \subseteq A$, we have $\varphi_{k, A}(y) \leq$ $\varphi_{k, A}(y+d)$. Since $y$ and $d$ are arbitrary and $0 \in \mathscr{D}\left(x_{0}\right)$, the equality in (i) holds.

Next, we get from $u \in W(x)$ that $F(u)+q(x, u) k+\mathscr{D}(x) \supseteq F(x)$ and thus

$$
F(u)+q(x, u) k+\mathscr{D}\left(x_{0}\right) \supseteq F(x) .
$$

By the properties of the scalarization function $\varphi_{k, A}$, we have

$$
\begin{aligned}
m(u)+q(x, u) & =q(x, u)+\inf \left\{\varphi_{k, A}(v+d): v \in F(u), d \in \mathscr{D}\left(x_{0}\right)\right\} \\
& =\inf \left\{\varphi_{k, A}(v+q(x, u) k+d): v \in F(u), d \in \mathscr{D}\left(x_{0}\right)\right\} \\
& \leq \inf \left\{\varphi_{k, A}(y): y \in F(x)\right\}=m(x) \quad \text { (by (3.10) }
\end{aligned}
$$

verifying inequalities in (ii). Observe that (iii) could be proved by using the same arguments in the proof of (ii).

Now, we are ready to formulate and prove a new version of Ekeland's variational principle in set optimization with domination structure.

Theorem 3.1. (EVP with the efficient and lower-set-less binary relation). Let the standing assumptions (H1)-(H4) hold. Assume that the following conditions hold:

(H5) (boundedness condition) $F$ is m-bounded from below on $W_{0}$ in the sense that there exists a finite number $\tau$ such that

$$
\forall x \in W_{0}, m(x) \geq \tau,
$$

where $m$ is the marginal function introduced in (2.4).

(H6) (lower-semicontinuity condition) $F$ is $<_{\mathscr{D}}^{l, e}$-decreasinglyforward-lower-semicontinuous on $W_{0}$ introduced in Definition 3.1.

Then there exists $x_{*} \in X$ satisfying

(i) $F_{x_{0}}\left(x_{*}\right) \leq \stackrel{\text { D,e }}{l,} F_{x_{0}}\left(x_{0}\right)$;

(ii) $\forall x \in X \backslash\left\{x_{*}\right\}, F_{x_{*}}(x) \underline{\leq}_{\mathscr{D}}^{l, e} F_{x_{*}}\left(x_{*}\right)$.

Obviously, (i) implies that $F\left(x_{*}\right) \leq_{\mathscr{D}}^{l, e} F\left(x_{0}\right)$ and

$$
q\left(x_{0}, x_{*}\right) \leq m\left(x_{0}\right)-\inf _{u \in W_{0}} m(u) \leq m\left(x_{0}\right)-\tau .
$$

The conclusion in (ii) means that $x_{*}$ is a strict $\leq_{\mathscr{D}}^{l, e}$-minimizer of the perturbed map $F_{*}$ introduced in (3.1).

Proof. We shall construct inductively a generalized Picard sequence which will satisfy all the conclusions of the theorem.

Start with the given element $x_{0}$. If $W\left(x_{0}\right)=\left\{x_{0}\right\}$, then $x_{0}$ satisfies all conclusions of the theorem. Set $x_{*}=x_{0}$ to complete the proof. Otherwise, set $\alpha_{0}:=\inf \left\{m(x): x \in W_{0}\right.$. By 
Proposition 3.3 (ii), we have $\alpha_{0}<m\left(x_{0}\right)<+\infty$. The boundedness assumption (H5) ensures that $\alpha_{0} \geq \tau$. We could find $x_{1} \in W_{0} \backslash\left\{x_{0}\right\}$ such that

$$
\alpha_{0} \leq m\left(x_{1}\right)<\frac{1}{2}\left(\alpha_{0}+m\left(x_{0}\right)\right)<m\left(x_{0}\right) .
$$

If $W\left(x_{1}\right)=\left\{x_{1}\right\}$, then $x_{1}$ satisfies all conclusions of the theorem. Set $x_{*}=x_{1}$ to complete the proof. Otherwise, set $W_{1}:=W\left(x_{1}\right)$ and $\alpha_{1}:=\inf \left\{m(x): x \in W_{1}\right\}$. We have $\alpha_{1}<m\left(x_{1}\right)$. Choose $x_{2} \in W_{1} \backslash\left\{x_{1}\right\}$ such that

$$
\alpha_{1} \leq m\left(x_{2}\right)<\frac{1}{2}\left(\alpha_{1}+m\left(x_{1}\right)\right)<m\left(x_{1}\right)
$$

Suppose that $x_{0}, x_{1}, \ldots, x_{n}$ were chosen. If $W\left(x_{n}\right)=\left\{x_{n}\right\}$, then $x_{n}$ satisfies all conclusions of the theorem. Set $x_{*}=x_{n}$ to complete the proof. Otherwise, set $W_{n}:=W\left(x_{n}\right)$ and $\alpha_{n}:=$ $\inf \left\{m(x): x \in W_{n}\right\}$. We have $\alpha_{n}<m\left(x_{n}\right)$. Choose $x_{n+1} \in W_{n} \backslash\left\{x_{n}\right\}$ such that

$$
\alpha_{n} \leq m\left(x_{n+1}\right)<\frac{1}{2}\left(\alpha_{n}+m\left(x_{n}\right)\right)<m\left(x_{n}\right) .
$$

It remains to justify the conclusions of the theorem in the case, where the sequence is infinite many terms. Obviously, $\left\{x_{n}\right\}_{n=0}^{+\infty}$ is a generalized Picard sequence of $W$ with positive steps. We claim that

$$
\sum_{n=0}^{\infty} q\left(x_{n}, x_{n+1}\right)<+\infty
$$

We have

$$
\forall n \in \mathbb{N} \cup\{0\}, F\left(x_{n+1}\right)+q\left(x_{n}, x_{n+1}\right) k+\mathscr{D}\left(x_{n}\right) \supseteq F\left(x_{n}\right) .
$$

By Proposition 3.1, we have

$$
F\left(x_{n+1}\right)+\sum_{i=0}^{n} q\left(x_{i}, x_{i+1}\right) k+\mathscr{D}\left(x_{0}\right) \supseteq F\left(x_{0}\right)
$$

By Proposition 3.3 (iii), we have

$$
m\left(x_{n+1}\right)+\sum_{i=0}^{n} q\left(x_{i}, x_{i+1}\right) \leq m\left(x_{0}\right) .
$$

By the boundedness assumption (H5), we have

$$
\sum_{i=0}^{n} q\left(x_{i}, x_{i+1}\right) \leq m\left(x_{0}\right)-\tau<+\infty
$$

Since $n$ was arbitrary, the claim is true. Then, for every $\varepsilon>0$, there is $N_{\varepsilon} \in \mathbb{N}$ such that for all $n \geq N_{\varepsilon}$ and for all $k \in \mathbb{N}$ we have

$$
q\left(x_{n}, x_{n+j}\right) \leq \sum_{i=n}^{n+j-1} q\left(x_{i}, x_{i+1}\right) \leq \sum_{i=n}^{\infty} q\left(x_{i}, x_{i+1}\right)<\varepsilon
$$

i.e., $\left\{x_{n}\right\}$ is a forward-Cauchy sequence in $(X, q)$. Taking into account the standing assumption (H4), it is forward-convergent. 
By Proposition 3.2, there exists a forward-limit $x_{*}$ of the sequence $\left\{x_{n}\right\}$, the nonempty intersection condition (3.5) holds. By Proposition 3.1 (W3), we have

$$
W\left(x_{*}\right) \in \bigcap_{n=0}^{\infty} W\left(x_{n}\right) .
$$

By Proposition 3.1 (W2), for every $n \in \mathbb{N} \cup\{0\}$, we have $m\left(x_{n+1}\right) \leq m\left(x_{n}\right)$. Since the decreasing sequence $\left\{m\left(x_{n}\right)\right\}$ is bounded from below by $\tau$ due to (H4), it is convergent to

$$
\underline{\alpha}=\lim _{n \rightarrow \infty} m\left(x_{n}\right)=\liminf _{n \rightarrow \infty} m\left(x_{n}\right) .
$$

By Proposition 3.1 (W3), for every $n \in \mathbb{N} \cup\{0\}$, we have $W_{n+1} \subseteq W_{n}$ and thus $\alpha_{n+1} \geq \alpha_{n}$. Since the sequence $\left\{\alpha_{n}\right\}$ is increasing and bounded from above by $m\left(x_{0}\right)$, it is converges to

$$
\bar{\alpha}=\lim _{n \rightarrow \infty} \alpha_{n}=\limsup _{n \rightarrow \infty} \alpha_{n} .
$$

Passing to limit the inequality in (3.11) as $n \rightarrow+\infty$, we have

$$
\underline{\alpha} \leq \frac{1}{2}(\underline{\alpha}+\bar{\alpha}) \leq \underline{\alpha}
$$

clearly implying that $\underline{\alpha}=\bar{\alpha}=\alpha$.

Next, we will show that $m\left(u_{*}\right)=\alpha$ for every $u_{*} \in W\left(x_{*}\right)$. Fix an arbitrary element $u_{*} \in$ $W\left(x_{*}\right)$. We get from (3.12) that for every $n \in \mathbb{I}, u_{*} \in W\left(x_{n}\right)$. It directly implies that $m\left(u_{*}\right) \geq$ $\inf \left\{m(u): u \in W\left(x_{n}\right)\right\}=\alpha_{n}$ and indirectly gives $m\left(u_{*}\right)+q\left(x_{n}, u_{*}\right) \leq m\left(x_{n}\right)$ due to Proposition 3.3. Passing to limit as $n \rightarrow+\infty$, we have $m\left(u_{*}\right) \geq \bar{\alpha}$ and $m\left(u_{*}\right) \leq \underline{\alpha}$. Since $\underline{\alpha}=\bar{\alpha}=\alpha$, $m\left(u_{*}\right)=\alpha$. Since $u_{*}$ was arbitrary, the claim is proved.

Finally, we show that $W\left(x_{*}\right)=\left\{x_{*}\right\}$ which is equivalent to (ii). Assume that $u_{*} \in W\left(x_{*}\right)$. Since we always have $x_{*} \in W\left(x_{*}\right)$, we have $m\left(u_{*}\right)=m\left(x_{*}\right)$. By Proposition 3.3, we get from $u_{*} \in W\left(x_{*}\right)$ that $m\left(u_{*}\right)+q\left(x_{*}, u_{*}\right) \leq m\left(x_{*}\right)$. Therefore, $q\left(x_{*}, u_{*}\right)=0$ which verifies that $u_{*}=$ $x_{*}$.

Remark 3.1. (new features in Theorem 3.1). (a) The boundedness condition in (H5) is equivalent to

$$
F\left(W_{0}\right) \cap(\tau k-A)=\emptyset .
$$

Such a map is

$$
F(x)= \begin{cases}{[0,+\infty) \times\{-x\}} & \text { if } x>0 \\ {[0,+\infty) \times[0,+\infty)} & \text { if } x=0, \\ \{x\} \times[0,+\infty) & \text { if } x<0\end{cases}
$$

This map is not $C$-quasibounded from below in the sense that here is a bounded subset $M$ such that $F\left(W_{0}\right) \subset M+C$ for some cone $C$ of $Y$.

(b) The image space $Y$ is extended to real linear spaces and use the so-called $k$-vectorial closedness as in [25].

(c) The quasimetric space $(X, q)$ is not necessarily forward-complete; condition (H4) is sufficient to establish variational principles of Ekeland-type.

(d) The $<_{\mathscr{D}}^{l, e}$-decreasingly lower-semicontinuity is further weaken this kind of assumption in variational principles of Ekeland-type. 
Remark 3.2. (comparisons with the existing results). (a) When $F=f: X \rightarrow Y$ is a singlevalued function, the new variational principle is better than [7, Theorem 3.1] and [25, Theorem 4.1(ii)] in several areas: (1) we do not assume that domination sets are closed and convex cones; (2) we do not assume that $f$ is quasibounded from below; and (3) we weaken the decreasing lower-semicontinuity.

(b) When $\mathscr{D}(x) \equiv K$ is a constant domination structure, where $K$ is close, convex and pointed cone in $Y$, the new variational principle improves [30, Theorem 3.1]. We do not assume that $F$ is $K$-lower-semicontinuous in the sense that for every $y \in Y$ the set $\{x \in X: F(x) \cap(y-K)\}$ is a closed set. It is important to mention that this assumption ensures that sets $W\left(x_{n}\right)$ are closed so that the Cantor theorem could be applied and that we do not use the Cantor theorem in our approach; indeed, we find the weakest assumption on the continuity behavior of $F$ such that the nonempty intersection in the Cantor theorem is still valid. We do not assume that $F$ is $K$-quasibounded; it is called $K$-bounded in the mentioned paper.

(c) When $Y=\mathbb{R}, \mathscr{D}(x) \equiv \mathbb{R}_{+}$, and $F=\varphi: X \rightarrow \mathbb{R}$, the new variational principle uses a weaker form of decreasingly lower-semicontinuity in comparison with Lemma 2.1.

\section{Applications to CHANGing JOBS}

4.1. Why changing jobs can be hard. The process of career change is not easy. Workers usually underestimate the process of career change and have not all the skills and expertise necessary for career change. They learn to change careers by doing it. We can make the distinction between three main categories of workers. "There is the type who settles for staying where they are, whether they are happy or not. The complete opposite of this is the type of person who never lets herself get tied down and never lets anyone else take control of her career. The final category fits in somewhere in the middle and contains the bulk of people- those who want a career change but are unable to do so because they are not mentally prepared for it." See [15].

Changing jobs is at the core of the main career development theories, including, among so many, Holland theory of vocational types (1985), social cognitive career theory [39] which is based on Bandura's social cognitive theory [2,3], developmental self-concept theory [51], career construction theory [44], matching theory [53], careership theory [26], work adjustment theory [17], happenstance theory [37], Gottfredson's theory of circumscription and compromise [24], etc. For a survey on career theories see [1, 40].

Our (VR) variational rationality approach of human dynamics [46, 47, 48, 49, 50] can allow to build (this must be done elsewhere) a comprehensive career development theory which is able to unify a lot of these approachs, including,

i) a proximal theory of changing job which explains why and how a worker can make a worthwhile move including a succession of stages within the current period, changing from doing a job in the previous period to doing an other job in the current period, and

ii) a distal theory of career development which modelizes a worthwhile transition following a succession of worthwhile moves within a succession of periods, ending in a final desired job or, much before, in a trap.

Here our goal is more mathematically oriented. Using the main VR concept of worthwhile move $[46,47,48,49,50]$, we can give an intuitive answer to the initial question "why changing job can be hard?". This happens as long as motivation to change job is not high enough with 
respect to resistance to change job. That is, when changing job is not a worthwhile move. Then, allowing a worker to make a succession of worthwhile moves to change from doing one job to doing an other job along a succession of periods, we show how the Ekeland variational principle for set-valued mappings with set-less relations gives sufficient conditions for the existence of traps which represent the ends of career development processes.

4.2. The variational rationality approach of human dynamics. Human dynamics. The (VR) variational rationality approach $[46,47,48,49,50]$ models and unifies a lot of different models of human dynamics which appeared in behavioral sciences (economics, management sciences, psychology, sociology, political sciences, decision theory, game theory, artificial intelligence, etc.). This dynamical approach considers entities (an agent, an organization or several interacting agents), which are, at the beginning of the story, in an undesirable initial position, and are, most of the time, unable, within the current period, to do a move which will allow them to reach, within this period, a final desired position. To hope to do so, they must accept to follow a transition, that is, a succession of moves (changes or stays), within a succession of periods. Hence, the goal of this approach is to examine transition problems where entities try to find, build and follow an acceptable and feasible transition which makes them able to overcome a lot of intermediate obstacles, difficulties and resistance to change, with not too much intermediate sacrifices and high enough intermediate satisfactions. This will help them to sustain motivation to move and persevere moving until reaching the final desired position. This VR approach admits a lot of variants, based on the same short list of general principles and concepts. The main concepts refer to moves (changes or stays), utility and costs of a move, a worthwhile move, worthwhile transitions made of a succession of worthwhile moves, stationary and variational traps. The core of the variational rationality approach of human dynamics $[46,47,48,49,50]$ is to give a definition of a worthwhile move.

A move $x \curvearrowright y$ is a change if $y \neq x$ and a stay if $y=x$. Within a period a stay refers to an exploitation phase, a temporary habit (doing the same bundle of activities in the previous and the current periods), an emergent routine, a rule, or a norm, while a change refers to an exploration phase, a learning and innovation process, building new capabilities, forming and breaking an habit or a routine, etc.

Advantages to move (change rather than stay). Let $g(x) \in R$ be the utility of doing again a bundle of activities $x$ in the current period, which is supposed to be equal to the utility of doing this bundle of activities $x$ for the first time. Let $g(y) \in R$ be the utility of doing for the first time the bundle of activities $y$ in the current period. Suppose that the agent has done the bundle of activities $x \in X$ in the previous period. Then, within the current period, $A(x, y)=g(y)-g(x) \geq 0$ represents advantages to move (change rather than stay at the status quo $x$ ) between doing again the bundle of activities $x \in X$ in the current period and doing the bundle of activities $y \in X$ in the current period. Let $g^{*}=\sup \{g(y), y \in X\}<+\infty$ be the highest utility the agent can hope to reach (his aspiration level). Then, $f(y)=g^{*}-g(y) \geq 0$ is the unsatisfaction feeling (frustration level) of the agent for not doing his best (not enjoying his highest level of utility within the period). This implies that $A(x, y)=g(y)-g(x)=f(x)-f(y)$.

Inconveniences to move (change rather than stay). They are the difference $I(x, y)=$ $C(x, y)-C(x, x) \geq 0$ between costs $C(x, y) \in R_{+}$of becoming able to do and do the chosen bundle of activities $y \neq x$ in the current period and costs $C(x, x) \in R_{+}$of becoming able to do 
again and do the previous bundle of activities $x$ in the current period. We can suppose, to simplify, that doing a job $z \in R_{+}$within a given period is identical to doing a bundle of activities $z={ }^{\text {def }}[0, z]$ where $[0, z]$ is an interval in $R_{+}$. Then, moving from having done the bundle of activities $x={ }^{\operatorname{def}}[0, x]$ in the previous period to do the bundle of activities $y^{\text {def }}=[0, y]$ in the current period requires,

i) if $y>x$, to start doing $y-x>0$ activities and to continue doing $x \geq 0$ activities,

ii) if $y<x$, to stop doing $x-y>0$ activities and to continue doing $y \geq 0$ activities, and

iii) if $y=x$, to continue doing $x \geq 0$ activities.

Let $\rho_{\backslash}>0, \rho_{=}>0$ and $\rho_{+}>0$ be the costs of being able to stop doing, being able to continue doing and do, being able to start doing and do one activity. Then,

$C(x, y)=\rho_{=} x+\rho_{+}(y-x)$ if $y \geq x$ and $C(x, y)=\rho_{=} y+\rho_{\backslash}(x-y)$ if $y<x$ model costs of moving from having done the job $x={ }^{d e f}[0, x]$ in the previous period to do the job $y={ }^{d e f}[0, y]$ in the current period. Costs to continue doing the job $x$ in the current period are $C(x, x)=\rho_{=} x$. To save space suppose that $\rho_{=}=0$. Then, $C(x, x)=0$, and inconveniences to move are

$$
I(x, y)=C(x, y)=\left\{\begin{array}{l}
\rho_{+}(y-x), \text { if } y \geq x \\
\rho_{\backslash}(x-y), \text { if } y \leq x
\end{array}\right\} \geq 0 .
$$

This defines a quasi-distance.

Payoff to move $x \curvearrowright y$. It represents either,

i) the net level of utility remaining after doing a move, $P(x, y)=g(y)-\xi C(x, y)$. It is the difference between the utility $g(y)$ of doing the new (or the same) bundle of activities $y$ and the costs of moving $C(x, y)$, where $\xi>0$ weights these costs of moving.

ii) the total level of dis-utility remaining after a move $Q(x, y)=f(y)+\xi C(x, y)$. It is the sum of the remaining unsatisfaction $f(y)$ of failing to reach the aspiration level of the worker after doing the new (or the same) bundle of activities $y$ and the costs of moving $C(x, y)$.

Motivation to move. It represents the utility of advantages to move $M(x, y)=U[A(x, y)]=$ $U[g(y)-g(x)]$.

Resistance to move. It refers to the dis-utility of inconveniences to move $R(x, y)=D[I(x, y)]=$ $D[C(x, x)-C(x, x)]$.

Worthwhile balance. $B_{\xi}(x, y)=M(x, y)-\xi R(x, y)$ is the balance between motivation and resistance to move (change rather stay).

Worthwhile move. A non negative balance $B \xi(x, y)=M(x, y)-\xi R(x, y) \geq 0$ defines a worthwhile move (change rather than stay). Then, a move $x \curvearrowright y$ is worthwhile if the utility $U[g(y)-g(x)]$ of advantages to move $A(x, y)=g(y)-g(x)>0$ is high enough with respect to the dis-utility $D[C(x, y)-C(x, x)]$ of inconveniences to move $I(x, y)=C(x, y)-C(x, x)$, that is, if $U[A(x, y)] \geq \xi D[I(x, y)]$, where $\xi>0$ is a worthwhile to move ratio. Then, a move is worthwhile if motivation to change rather than to stay $M(x, y)$ is "high enough" with respect to resistance to change rather than to stay $R(x, y)$. This definition can model a lot of variants for the definitions of motivation and resistance to move, because one hundred theories/aspects of motivations exist in psychology, and resistance to move includes a lot of different aspects (see $[46,47,48,49,50])$.

The linear formulation. In this paper, we consider the linear case where motivation and resistance to move are identical to advantages and inconveniences to move, that is, when 
$U[A(x, y)]=A(x, y)=g(y)-g(x)=f(x)-f(y)$ and $D[I(x, y)]=I(x, y)=C(x, y)-C(x, x)$, for all $x, y \in X$. In this context a worthwhile balance can be written as,

$B_{\xi}(x, y)=A(x, y)-\xi I(x, y)$; that is, the difference between advantages and inconveniences to move,

$B_{\xi}(x, y)=g(y)-g(x)-\xi I(x, y)=f(x)-f(y)-\xi I(x, y)$, or,

$B_{\xi}(x, y)=P(x, y)-P(x, x)$, i.e., the difference between the net utility to do a change and the net utility to stay, or,

$B_{\xi}(x, y)=Q(x, x)-Q(x, y)=P(x, y)-P(x, x)$, i.e., the difference between the gross remaining unsatisfaction to do a change and the gross remaining unsatisfaction to stay.

Then, a worthwhile move $x \curvearrowright y$ is such that,

a) advantages to move (change rather than stay) are high enough with respect to inconveniences to move, i.e., $A(x, y) \geq \xi I(x, y)$, that is, $g(y)-g(x)=f(x)-f(y) \geq \xi I(x, y)$, or

b) "to be increased" payoffs to move increase, i.e., $P(x, y) \geq P(x, x)$, or

c) "to be decreased" payoffs to move decrease, that is, $Q(x, y) \leq Q(x, x)$, or

d) its worthwhile balance is non negative, i.e., $B_{\xi}(x, y)=g(y)-g(x)-\xi I(x, y)=f(x)-$ $f(y)-\xi I(x, y) \geq 0$.

A stationary trap. A stationary trap $x_{*} \in X$ is such that it is not worthwhile to move from $x_{*}$ to any $y \neq x_{*}$, i.e., $M\left(x_{*}, y\right)<\xi R\left(x_{*}, y\right)$ for any $y \neq x_{*}$.

That is, in the linear case where $U[A(x, y)]=A(x, y)$ and $D[I(x, y)]=I(x, y)$ for all $x, y \in X$, a stationary trap $x_{*}$ is such that

$A\left(x_{*}, y\right)<\xi I\left(x_{*}, y\right)$ for all $y \neq x_{*}$, that is,

$A\left(x_{*}, y\right)=g(y)-g\left(x_{*}\right)=f\left(x_{*}\right)-f(y)<\xi I\left(x_{*}, y\right)=\xi[C(x, y)-C(x, x)]$ for all $y \neq x_{*}$,

or each of the following equivalent conditions,

$P\left(x_{*}, y\right)<P\left(x_{*}, x_{*}\right)$, or $Q\left(x_{*}, y\right)>Q\left(x_{*}, x_{*}\right)$, or $B \xi\left(x_{*}, y\right)<0$ for all $y \neq x_{*}$.

A worthwhile transition. It is a succession of worthwhile moves (changes or stays).

A variational trap $x_{*}$ is, both, i) an aspiration point, worthwhile to approach and reach, following a worthwhile transition which starts from an initial position $x_{0}$ and, ii) a stationary trap not worthwhile to leave. Then, in the linear case and if a worthwhile transition includes only one worthwhile move, it satisfies the two conditions,

ii) $g\left(x_{*}\right)-g\left(x_{0}\right)=f\left(x_{0}\right)-f\left(x_{*}\right) \geq \xi I\left(x_{0}, x_{*}\right)$,

iii) $g(y)-g\left(x_{*}\right)=f\left(x_{*}\right)-f(y)<\xi I\left(x_{*}, y\right)$ for all $y \neq x_{*}$,

or the two conditions,

ii) $P\left(x_{0}, x_{*}\right) \geq P\left(x_{0}, x_{0}\right)$, i.e., $Q\left(x_{0}, x_{*}\right) \leq Q\left(x_{0}, x_{0}\right)$,

iii) $P\left(x_{*}, y\right)<P\left(x_{*}, x_{*}\right)$, i.e., $Q\left(x_{*}, y\right)>Q\left(x_{*}, x_{*}\right)$ for all $y \neq x_{*}$,

or equivalently, the two conditions,

ii) $B \xi\left(x_{0}, x_{*}\right) \geq 0$,

iii) $B_{\xi}\left(x_{*}, y\right)<0$ for all $y \neq x_{*}$.

Existence of variational traps: Ekeland theorem in a quasi metric space. The VR approach $([46,47,48,49,50])$ showed that the formulation of the Ekeland variational principle [20] in a quasi metric space gives sufficient conditions for the existence of variational traps. It considers, both, the usual minimizing formulation given in mathematics (cost minimization) and its maximizing variant better adapted to behavioral sciences (utility or satisfaction). 
Ekeland theorem (minimizing case). Let $(X, q)$ be a left-complete quasi metric space where $q(.,):.(x, y) \longmapsto q(x, y) \in R_{+}$is a quasi metric. Let $f():. x \in X \longmapsto f(x) \in \bar{R}$ be a "to be decreased" payoff function, which is proper, bounded below, 1-1.s.c and $\varepsilon>0$. Let $f_{*}=$ $\inf \{f(z), z \in X\}>-\infty$. Suppose that $x_{0} \in X$ satisfies $f\left(x_{0}\right) \leq f_{*}+\varepsilon$.

Let $F_{x}():. y \in X \longmapsto F_{x}(y)=f(y)+(\varepsilon / \lambda) q(x, y) \in R$ be a perturbation function of $f($.$) at x$. Then, for any $\lambda>0$, it exists $x_{*} \in X$ such that

i) $q\left(x_{0}, x_{*}\right) \leq \lambda$,

ii) $f\left(x_{*}\right)+(\varepsilon / \lambda) q\left(x_{0}, x_{*}\right) \leq f\left(x_{0}\right)$, that is, $F_{x_{0}}\left(x_{*}\right) \leq F_{x_{0}}\left(x_{0}\right)$ (perturbation function formulation), i.e.,

$$
f\left(x_{0}\right)-f\left(x_{*}\right) \geq(\varepsilon / \lambda) q\left(x_{0}, x_{*}\right) \text {, that is, } A\left(x_{0}, x_{*}\right) \geq(\varepsilon / \lambda) I\left(x_{0}, x_{*}\right) \text { (VR formulation) }
$$

iii) $f(y)+(\varepsilon / \lambda) q\left(x_{*}, y\right)>f\left(x_{*}\right)$, that is, $F_{x_{*}}(y)>F_{x_{*}}\left(x_{*}\right)$ (perturbation function formulation), i.e.,

$$
\begin{aligned}
& f\left(x_{*}\right)-f(y)<(\varepsilon / \lambda) q\left(x_{*}, y\right) \text {, that is, } A\left(x_{*}, y\right)<(\varepsilon / \lambda) I\left(x_{*}, y\right) \text { (VR formulation) } \\
& \text { for all } y \in X \backslash\left\{x_{*}\right\} .
\end{aligned}
$$

Ekeland theorem (maximizing case). Let $g():. x \in X \longmapsto g(x) \in \bar{R}$ be a "to be increased" payoff function, which is proper, bounded above, r-u.s.c and $\varepsilon>0$. Let $g^{*}=\sup \{g(z), z \in X\}<$ $+\infty$.

Suppose that $x_{0} \in X$ satisfies $g\left(x_{*}\right) \geq g^{*}-\varepsilon$.

Let $G_{x}():. y \in X \longmapsto G_{x}(y)=g(y)-(\varepsilon / \lambda) q(x, y) \in R$ be a perturbation function of $g($.$) at x$.

Then, for any $\lambda>0$, it exists $x_{*} \in X$ such that

i)' $q\left(x_{0}, x_{*}\right) \leq \lambda$,

ii)' $g\left(x_{*}\right)-(\varepsilon / \lambda) q\left(x_{0}, x_{*}\right) \geq g\left(x_{0}\right)$, that is, $G_{x_{0}}\left(x_{*}\right) \geq G_{x_{0}}\left(x_{0}\right)$ (perturbation function formulation), i.e., $g\left(x_{*}\right)-g\left(x_{0}\right) \geq(\varepsilon / \lambda) q\left(x_{0}, x_{*}\right)$, that is, $A\left(x_{0}, x_{*}\right) \geq(\varepsilon / \lambda) I\left(x_{0}, x_{*}\right)$ (VR formulation),

iii)' $g(y)-(\varepsilon / \lambda) q\left(x_{*}, y\right)<g\left(x_{*}\right)$, that is, $G_{x_{*}}(y)<G_{x_{*}}\left(x_{*}\right)$ (perturbation function formulation), i.e., $g(y)-g\left(x_{*}\right)<(\varepsilon / \lambda) q\left(x_{*}, y\right)$, that is, $A\left(x_{*}, y\right)<(\varepsilon / \lambda) I\left(x_{*}, y\right)$ (VR formulation), for all $y \in X \backslash\left\{x_{*}\right\}$.

The usual formulation of the Ekeland theorem in term of perturbation functions tells us that it exists $x_{*}$ which (see ii) or ii)') improves (decreases for the minimizing case and increases for the maximizing case) the perturbation function at $x_{0}$ and, (see iii) or iii)') optimizes strictly (minimizes or maximizes strictly) the perturbation function at $x_{*}$.

The VR approach interprets the Ekeland theorem (maximizing case) as follows. The "to be increased" payoff function $g($.$) is an utility function, g^{*}$ is an aspiration level (the maximum utility an agent can hope to reach), $\xi=\varepsilon / \lambda>0$ is a worthwhile to move ratio (threshold), $x \in X$ is the bundle of activities that an agent have done in the previous period, $y \in X$ is the bundle of activities that the agent wants to do in the current period, $A(x, y)=g(y)-g(x)$ represents an advantage to move from $x$ to $y, C(x, y)$ represents costs of moving from $x$ to $y, I(x, y)=C(x, y)-$ $C(x, x) \geq 0$ refers to inconveniences to move from $x$ to $y$, motivation to move is $M(x, y)=$ $U[A(x, y)]=A(x, y)$ and resistance to move is $R(x, y)=D[I(x, y)]=I(x, y)$. Finally the quasi metric $q(x, y)=I(x, y)$ modelizes inconveniences to move. 
Then, points i)', ii)', iii)' tell us that, i)' inconveniences to move from $x$ to $x_{*}$ must be low enough, ii)' it is worthwhile to directly move from $x$ to $x_{*}$ (the transition reduces to one worthwhile move), iii)' it is not worthwhile to leave $x_{*}$. Then, the maximizing version of the Ekeland theorem gives sufficient conditions for the existence of variational traps.

The VR interpretation of the minimizing version of the Ekeland theorem is similar, using an equivalent formulation of advantages to move. Considers (see above) the unsatisfaction function $f(x)=g^{*}-g(x) \geq 0$ which defines how much of the aspiration level $g^{*}$ the agent fails to satisfy. In this context, advantages to move represent the difference between unsatisfaction $f(x) \geq 0$ to stay and unsatisfaction to change $f(y) \geq 0$. That is, $A(x, y)=g(y)-g(x)=f(x)-f(y)$. Then, conditions ii) and iii) are identical to conditions ii)' and iii)', where,

ii) $f\left(x_{0}\right)-f\left(x_{*}\right)=g\left(x_{*}\right)-g\left(x_{0}\right) \geq(\varepsilon / \lambda) q\left(x_{0}, x_{*}\right)$ and,

iii) $f\left(x_{*}\right)-f(y)=g(y)-g\left(x_{*}\right)<(\varepsilon / \lambda) q\left(x_{*}, y\right)$ for all $y \in X \backslash\left\{x_{*}\right\}$.

The same interpretation follows.

4.3. The changing jobs problem in an unknown and changing context. In this last section we give an application to a changing city and job problem where a worker can be obliged to move from living in a city to living in an other city, in order to find a better job, when,

i) this worker (agent) have several goals,

ii) the importance of goals change (an instance of an adaptive decision making process modelized by a domination structure),

iii) deep uncertainty matters (robust decision making in a complex environment).

In this context the problem is not to maximize some utility, but to minimize regret.

4.3.1. Changing locations and jobs. This is a variant of a model of moving from one location (city) to an other location given in [49].

Do I change, do I stay? Consider a set of cities $X$. Let $x \in X$ be the city where a worker lives in a given period. Let $J(x)$ be a list of job offers in city $x$, that is, a list of jobs (bundle of working activities) that a worker can do and can apply for in city $x$ in this given period.

Consider a worker who lived and has done a job $i \in J(x)$ in city $x$ in the previous period. Suppose also that, in the current period, this worker balances between,

ii) change from living in city $x$ in the previous period to living in city $x^{\prime}$ in the current period, and do a job $j^{\prime} \in J\left(x^{\prime}\right)$ in city $x^{\prime}$ in the current period,

i) stay (live again) in city $x$ in the current period, and do this same job $i \in J(x)$ or a different job $j \in J(x)$ in city $x$ in this current period.

Then, in the current period, a change is $(i, x) \curvearrowright\left(j^{\prime}, x^{\prime}\right)$ and a stay is $(i, x) \curvearrowright(j, x)$.

The payoff space of vectorial pleasures $Y$. For simplification take $Y=R^{m}$ and $\Theta=R_{+}^{m}$. Consider a list which includes different kinds of pleasures $h=1,2, \ldots, m$ that a worker can get when living in a city and doing a job in this city within a period. Let

$$
g(j, x)=\left\{g^{h}(j, x), h=1,2, \ldots, m\right\} \in Y
$$

be the amounts of the different types of physiological, physical, material, financial, cognitive/mental, affective, and social pleasures $h=1,2, \ldots, m$ coming from living in city $x$ and doing the job $j \in J(x)$ in this city. Let $g^{* h}<+\infty$ be the maximal level of pleasure of type $h$ that a worker can hope to get within a period, coming from living in a city and doing a job in 
this city. Then, $f^{h}(j, x)=g^{* h}-g^{h}(j, x) \geq 0$ represents the different physiological, physical, material, financial, cognitive/mental, affective, and social unsatisfaction feelings coming from failing to reach each maximal level $g^{* h}, h=1, \ldots, m$, when living in city $x$ and doing the job $j \in J(x)$ in this city in the current period. Let $f(j, x)=\left\{f^{h}(j, x), h=1,2, \ldots, m\right\} \in Y$ be the vector of these unsatisfaction feelings of living in city $x$ and doing job $j \in J(x)$ in city $x$ in the current period.

The payoff space of vectorial pains $Y$. Like pleasures (utilities), pains (costs) can be physiological (effort), physical (effort), material (machines and tools), financial, cognitive/mental (effort), affective (feelings), and social. These pains represent different kinds of costs. They include costs of becoming able to do different activities and costs to do these activities within a period.

Suppose that, living in city $x$ in the previous period, a worker has done the job $i \in J(x)$ in this previous period. There are two cases:

i) either he changes, living in a new city $x^{\prime} \neq x$ and doing a different job $j^{\prime} \in J\left(x^{\prime}\right), j^{\prime} \neq i$, in the current period,

ii) or, he stays, living in the same city $x^{\prime}=x$ and doing the same or a different job $j \in J(x)$, $j=i$ or $j \neq i$, in the current period.

Costs to move from living in city $x$ in the previous period to be able to live and live in city $x^{\prime}$ in the current period are $C\left(x, x^{\prime}\right) \in \Theta=R_{+}^{m} \subset Y$.

Costs to be able to live again and live in city $x$ in the current period are $C(x, x) \in \Theta$.

Costs to become able to change jobs, moving from having done the job $i \in J(x)$ in city $x$ in the previous period to become able to do and do a new job $j^{\prime} \in J\left(x^{\prime}\right)$ in city $x^{\prime}$ in the current period are $K\left(i, j^{\prime}\right) \in \Theta \subset Y$.

Vectorial advantages to move (change rather than stay). Advantages to move (change rather than stay) represent the variations of the different kinds of pleasures between doing a different bundle of activities and doing again the same bundle of activities.

$A\left((j, x),\left(j^{\prime}, x^{\prime}\right)\right)=g\left(j^{\prime}, x^{\prime}\right)-g(j, x)=f(j, x)-f\left(j^{\prime}, x^{\prime}\right) \in Y$ from $f^{h}(j, x)=g^{* h}-g^{h}(j, x) \geq$ $0, h=1, \ldots, m$.

Vectorial inconveniences to move (change rather than stay). They are

$\mathbb{I}\left((j, x),\left(j^{\prime}, x^{\prime}\right)\right)=I\left(x, x^{\prime}\right)+H\left((i, j),\left(i, j^{\prime}\right)\right)$ where,

$I\left(x, x^{\prime}\right)=C\left(x, x^{\prime}\right)-C(x, x) \in \Theta \subset Y$ and $H\left((i, j),\left(i, j^{\prime}\right)\right)=K\left(i, j^{\prime}\right)-K(i, j) \in \Theta \subset Y$. For simplification we will suppose equal costs of changing jobs, that is, $K\left(i, j^{\prime}\right)=K(i, j)$ for all $i \in J(x), j \in J(x)$ and $j^{\prime} \in J\left(x^{\prime}\right)$. In this context inconveniences to move refer to inconveniences to move from one city to a new one, that is, $\mathbb{I}\left((j, x),\left(j^{\prime}, x^{\prime}\right)\right)=I\left(x, x^{\prime}\right) \in Y$.

In this paper we take the simple formulation $I\left(x, x^{\prime}\right)=C\left(x, x^{\prime}\right)-C(x, x)=q\left(x, x^{\prime}\right) k \in \Theta=$ $R_{+}^{m}$, where $k \in Y-\{0\}$ and $q(.,):.\left(x, x^{\prime}\right) \in X . X \longmapsto q\left(x, x^{\prime}\right) \in R_{+}$is a quasi metric. Then, $\mathbb{I}\left((j, x),\left(j^{\prime}, x^{\prime}\right)\right)=q\left(x, x^{\prime}\right) k$. The scalar $q\left(x, x^{\prime}\right) \in R_{+}$represents the total inconveniences of moving from a city to an other city. If $Y=R^{m}$, the vector $k=\left(k^{1}, \ldots, k^{l}, \ldots, k^{m}\right) \in \Theta, \Sigma_{l=1}^{m} k^{l}=1$ modelizes the shares of these total inconveniences to move which represent the size of each different kind of inconveniences to move which can be physiological (effort), physical (effort), material (machines and tools), financial, cognitive/mental (effort), affective (feelings), and social.

Vectorial worthwhile balance. It is the difference between vectorial advantages to move and vectorial inconveniences to move, 
$\mathscr{B}_{\xi}\left((j, x),\left(j^{\prime}, x^{\prime}\right)\right)=A\left((j, x),\left(j^{\prime}, x^{\prime}\right)\right)-\xi \mathbb{I}\left((j, x),\left(j^{\prime}, x^{\prime}\right)\right)$, i.e.,

$\mathscr{B}_{\xi}\left((j, x),\left(j^{\prime}, x^{\prime}\right)\right)=g\left(j^{\prime}, x^{\prime}\right)-g(j, x)-\xi_{q}\left(x, x^{\prime}\right) k \in \Theta=R_{+}^{m}$, or,

$\mathscr{B}_{\xi}\left((j, x),\left(j^{\prime}, x^{\prime}\right)\right)=f(j, x)-f\left(j^{\prime}, x^{\prime}\right)-\xi q\left(x, x^{\prime}\right) k \in \Theta$,

where $\xi \in R_{+}$is a scalar which weights the importance of inconveniences to move. For simplification we will take $\xi=1$.

Vectorial worthwhile move. Consider a worker who lived in city $x$ and do a job $i \in J(x)$ in this city in the previous period and balances, in the current period, between,

i) change, that is, choose to live in city $x^{\prime}$ and do a new job $j^{\prime} \in J\left(x^{\prime}\right)$ in city $x^{\prime}$ in the current period,

ii) stay, i.e., chooses to live again in city $x$ and do the same job $i \in J(x)$ or a different job $j \in J(x), j \neq i$, in city $x$ in the current period. Then, it is, ex ante, worthwhile to move (change rather than stay) if $A\left((j, x),\left(j^{\prime}, x^{\prime}\right)\right)=g\left(j^{\prime}, x^{\prime}\right)-g(j, x)=f(j, x)-f\left(j^{\prime}, x^{\prime}\right) \geq_{\Theta}$ $q\left(x, x^{\prime}\right) k=I\left(x, x^{\prime}\right)$, i.e.,

$f\left(j^{\prime}, x^{\prime}\right)+q\left(x, x^{\prime}\right) k \leq_{\Theta} f(j, x)$, or,

$g\left(j^{\prime}, x^{\prime}\right)-q\left(x, x^{\prime}\right) k \geq_{\Theta} g(j, x)$, that is,

$\mathscr{B}_{\xi}\left((j, x),\left(j^{\prime}, x^{\prime}\right)\right)=f(j, x)-f\left(j^{\prime}, x^{\prime}\right)-q\left(x, x^{\prime}\right) k \in \Theta$, or,

$\mathscr{B}_{\xi}\left((j, x),\left(j^{\prime}, x^{\prime}\right)\right)=g\left(j^{\prime}, x^{\prime}\right)-g(j, x)-q\left(x, x^{\prime}\right) k \in \Theta$.

4.3.2. When the importance of a worker's goals changes with the situation: non conic domination structures. Consider a worker who wants to minimize the amounts $v=\left(v^{1}, \ldots, v^{h}, \ldots, v^{m}\right) \in$ $Y=R_{+}^{m}$ of simultaneous and different pains which can be physiological, physical, material, financial, cognitive/mental, psychological, and social. Let us generalize, progressively, the vectorial relation $v \leq_{\Theta} y$ which means that "a vector of pains $v=\left(v^{1}, \ldots, v^{h}, \ldots, v^{m}\right) \in Y$ is better ("lower") than a vector of pains $y=\left(y^{1}, \ldots, y^{h}, \ldots, y^{m}\right) \in Y^{\prime}$ ". In this context the worker have several goals, each of them being to minimize a different kind of pains. These simultaneous goals can have the same or different importances for the worker and these importance are fixed or can change.

a) First usual case: the same and constant importance for all goals. Start with the usual case where $\Theta=R_{+}^{m}$ is the non negative orthant of $Y=R^{m}$. It is a constant cone which modelizes the fixed and equal importance of each pain/goal. Less of each pain being better than more, $v \leq_{\Theta} y$ iff $y-v \in \Theta$ that is, iff $v^{h} \leq y^{h}$, i.e., $y^{h}-v^{h} \geq 0$ for all $h=$ $1,2, \ldots, m$. In this context, $\Theta$ is the subset of pains "worse than" zero: if $v=0,0 \leq_{\Theta} y$ iff $y \in \Theta$, i.e., iff $y^{h} \geq 0$ for all $h=1,2, \ldots, m$. In this case each goal have the same importance because, for each pain, the agent requires less, without trading less of a pain against more (but not too much) of an other one. Then, the relation $v=f\left(j^{\prime}, x^{\prime}\right)+$ $q\left(x, x^{\prime}\right) k \leq_{\Theta} f(j, x)=y$ is equivalent to $y-v \in \Theta$. It defines a worthwhile move if its worthwhile balance is non negative, that is, if $\mathscr{B}_{\xi}\left((j, x),\left(j^{\prime}, x^{\prime}\right)\right)=f(j, x)-f\left(j^{\prime}, x^{\prime}\right)-$ $q\left(x, x^{\prime}\right) k \in \Theta$.

b) Second case: different and constant importance for each goal. Now consider a constant cone $D \supset \Theta$ instead of the non negative orthant $\Theta$ as a subset of pains "worse than" zero. In this setting trade-offs like "to prefer less of a pain against more (but not too much) of an other pain" are considered. For example, for two different kinds of pains 1 and 2 where it is more important to decrease pain 1 than pain $2, v=\left(v^{1}, v^{2}\right)$ is prefered to $y=\left(y^{1}, y^{2}\right)$, that is, $v \leq_{D} y$ if $v^{1}<v^{2}$ even if $y^{1}>y^{2}$ when $y^{1}-y^{2}$ is not too positive. 
In that case ([57]), $v \leq_{D} y$ is equivalent to $y-v \in D$. Then, the relation $v=f\left(j^{\prime}, x^{\prime}\right)+q\left(x, x^{\prime}\right) k \leq_{D} f(j, x)=y$ is equivalent to $y-v \in D$. It defines a worthwhile move if its worthwhile balance

$\mathscr{B}_{\xi}\left((j, x),\left(j^{\prime}, x^{\prime}\right)\right) \in D$.

c) Third case: the importance of different goals are different and change. A domination structure ([57]) modelizes this situation where $v \leq_{\mathscr{D}(r)} y$ iff $y-v \in D(r), \mathscr{D}(r)$ being a variable cone which changes with the reference point $r$. If $r=x$,

$v=f\left(j^{\prime}, x^{\prime}\right)+q\left(x, x^{\prime}\right) k \leq \mathscr{D}(x) f(j, x)=y$ is equivalent to $y-v \in D(x)$, that is, $\mathscr{B}_{\xi}((j, x)$, $\left.\left(j^{\prime}, x^{\prime}\right)\right) \in \mathscr{D}(x)$.

d) Fourth case: our paper. Relative to a vectorial minimization problem, we consider a non conic domination structure which defines for each $x \in X$ a subset $\mathscr{D}(x) \subset Y$ of pains "worse than" $x$, such that $0 \in \mathscr{D}(x)$ and $\mathscr{D}(x)+\mathscr{D}(x) \subset \mathscr{D}(x)$ for each $x \in X$. This set $\mathscr{D}(x)$ is not a cone.

4.3.3. When deep uncertainty matters: search for a robust solution. Consider a non conic domination structure $\{D(x), x \in X\}$.

Deep uncertainty. Here we embed the problem of changing jobs in a preliminary problem of choosing to live again in the same city or to live in an other city. Then, comes the choice of choosing a job in a given city.

Let $J(x) \subset J$ be the set of jobs a worker can apply for each period, when living in city $x$ in a given period. Suppose also that a job lasts for one period. Then, the next period, a worker living in city $x$ and doing the job $i \in J(x)$ must apply for the same or a new job $j \in J(x)$ in city $x$ or an other job $j^{\prime} \in J\left(x^{\prime}\right)$ in an other city $x^{\prime}$. This implies that, next period, when a worker balances between living again in city $x$ and living in a new city $x^{\prime}$, that is, before choosing to make a stay $x \curvearrowright x^{\prime}=x$ or change $x \curvearrowright x^{\prime}, x^{\prime} \neq x$, the worker does not know which job he will get within the list of jobs $J(x)$ he can apply for if he lives again in city $x$ and which job he will get within the list of jobs $J\left(x^{\prime}\right)$ he can apply for if he lives in the new city $x^{\prime}$. Then, he must do a choice between stay and change in a context of deep uncertainty. Hence his goal is not to maximize his pleasures and to minimize his pains, but to do not regret his choice of location.

Definition of a robust worthwhile move. In this context of deep uncertainty, it will be worthwhile for a worker to move from city $x$ in the previous period to city $x^{\prime}$ in the current period if FOR EVERY job $j \in J(x)$ that the worker can do again in city $x$ in the current period, IT EXISTS a job $j^{\prime} \in J\left(x^{\prime}\right)$ that the worker can do in city $x^{\prime}$ in the current period such that it is worthwhile to move from doing again the job $j \in J(x)$ in city $x$ in the current period to start doing the job $j^{\prime} \in J\left(x^{\prime}\right)$ in city $x^{\prime}$ in the current period, i.e., if

$f\left(j^{\prime}, x^{\prime}\right)+q\left(x, x^{\prime}\right) k \leq_{D(x)} f(j, x)(*)$.

Let $F(x)=\{f(j, x), j \in J(x)\}$ be the subset of pains of the worker when living in city $x$ and doing a job $j \in J(x)$ in city $x$ in the current period. Let $F\left(x^{\prime}\right)=\left\{f\left(j^{\prime}, x^{\prime}\right), j^{\prime} \in J\left(x^{\prime}\right)\right\}$ be the subset of pains of the worker when living in city $x^{\prime}$ and doing a job $j^{\prime} \in J\left(x^{\prime}\right)$ in city $x^{\prime}$ in the same period. Then, $\left({ }^{*}\right)$ shows that it is worthwhile for a worker to move from city $x$ in the previous period to city $x^{\prime}$ in the current period if

$$
A=F_{x}\left(x^{\prime}\right)=F\left(x^{\prime}\right)+q\left(x, x^{\prime}\right) k \leq_{D(x)}^{l} B=F_{x}(x)=F(x)(* *) .
$$

Proof. Inequality $A \leq_{D(x)}^{l} B$ (**) is true if and only if $\forall b=f(j, x) \in B, \exists a=f\left(j^{\prime}, x^{\prime}\right)+$ $q\left(x, x^{\prime}\right) k \in A$ such that $a \leq_{D(x)} b$, i.e., $b=a+\theta$, that is, if for all $\mathrm{j} \in J(x)$, it exists $j^{\prime} \in J\left(x^{\prime}\right)$ such 
that $b=f(j, x)=f\left(j^{\prime}, x^{\prime}\right)+q\left(x, x^{\prime}\right) k+\theta=a+\theta$, with $\theta \in D(x)$, i.e., $f\left(j^{\prime}, x^{\prime}\right)+q\left(x, x^{\prime}\right) k \leq_{D(x)}$ $f(j, x)\left(^{*}\right)$. Then, a robust worthwhile move from city $x$ to city $x^{\prime}$ is such that $F\left(x^{\prime}\right)+q\left(x, x^{\prime}\right) k+$ $D(x) \supset F(x)$.

Remark 4.1. As a consequence, the definition of a non robust worthwhile move is the following: it is not worthwhile (in a robust sense) to move from city $x$ in the previous period to city $x^{\prime}$ in the current period if IT EXISTS a job $j^{\$} \in J(x)$ that the worker can do again in city $x$ in the current period such that FOR EVERY job $j^{\prime} \in J\left(x^{\prime}\right)$ that the worker can do in city $x^{\prime}$ in the current period it is not worthwhile to move from doing again the job $j^{\$} \in J(x)$ in city $x$ in the current period to start doing the job $j^{\prime} \in J\left(x^{\prime}\right)$ in city $x^{\prime}$ in the current period, i.e., if $f\left(j^{\prime}, x^{\prime}\right)+q\left(x, x^{\prime}\right) \xi \npreceq \mathscr{D}(x) f\left(j^{\$}, x\right)$ for every job $j^{\prime} \in J\left(x^{\prime}\right)$. This definition of a non robust worthwhile move makes sense if we say that there is some probability (even small) that it is not worthwhile to move from city $x$ to city $x^{\prime}$.

\subsection{Our result. The existence of robust traps in unknown environments and changes in} the importance of goals. Result. Behavioral interpretation of Theorem 3.1.

Under the list of hypothesis given in theorem 3.1, given a city $x_{0}$, it exists a city $x_{*} \in X$ such that,

i) it is worthwhile (in a robust sense) for a worker to move from living and working in city $x_{0}$ to live and work in city $x_{*}$ and, then, change jobs,

ii) for each $x \neq x_{*}$ it is not worthwhile (in a robust sense) to move from city $x_{*}$ to any other city in order to change jobs.

\section{Acknowledgments}

The first author wishes to thank the AMSE, Aix-Marseille University for the very warm hospitality and providing the excellent facilities during his visit at Chateau Lafarge, AMSE, AixMarseille University.

\section{REFERENCES}

[1] J.A. Athanasou, R. Van Esbroeck, International Handbook of Career Guidance, Springer, 2008.

[2] A. Bandura, Social learning theory, Englewood Cliffs, NJ, Prentice-Hall, 1977.

[3] A. Bandura, Self-efficacy: The exercise of control, New York, Freeman, 1997.

[4] T.Q. Bao, S. Cobzaş, A. Soubeyran, Variational principles, completeness and the existence of traps in behavioral sciences, Ann. Oper. Res. 269 (2018), 53-79.

[5] T.Q. Bao, P.Q. Khanh, A. Soubeyran, Variational principles with generalized distances and the modelization of organizational change, Optimization 65 (2016), 2049-2066.

[6] T.Q. Bao, B.S. Mordukhovich, A. Soubeyran, Variational analysis in psychological modeling, J. Optim. Theory Appl. 164 (2015), 290-315.

[7] T.Q. Bao, B.S. Mordukhovich, A. Soubeyran, Fixed points and variational principles with applications to capability theory of wellbeing via variational rationality, Set-Valued Var. Anal. 23 (2015), 375-398.

[8] T.Q. Bao, B.S. Mordukhovich, Necessary nondomination conditions for set and vector optimization with variable structures, J. Optim. Theory Appl. 162 (2014), 350-370.

[9] T.Q. Bao, G. Eichfelder, B. Soleimani, C. Tammer, Ekeland's variational principle for vector optimization with variable ordering structure, J. Convex Anal. 24 (2017), 393-415.

[10] T.Q. Bao, A. Soubeyran, Variational analysis in cone pseudo-quasimetric spaces and applications to group dynamics, J. Optim. Theory Appl. 170 (2016), 458-475.

[11] T.Q. Bao, M.A. Théra, On extended versions of Dancs-Hegedüs-Medvegyev's fixed-point theorem, Optimization 66 (2017), 875-887. 
[12] K. Bergstresser, P.L. Yu, Domination structures and multicriteria problems in N-person games. Game theory and conflict resolution, I, Theory Decis. 8 (1977), 5-48.

[13] K. Bergstresser, A. Charnes, P.L. Yu, Generalization of domination structures and nondominated solutions in multicriteria decision making, J. Optim. Theory Appl. 18 (1976), 3-13.

[14] G.Y. Chen, X. Huang, X. Yang, Vector Optimization, Set-Valued and Variational Analysis, Springer, Heidelberg, 2005.

[15] N. Chitakasem, The psychology of career change - Why is it so hard? Position Ignition, Tuesday June 28, 2016 at $12: 00$.

[16] S. Dancs, M. Hegedüs, P. Medvegyev, A general ordering and fixed-point principle in complete metric space, Acta Scientiarum Mathematicarum (Szeged), 46 (1983), 381-388.

[17] R.V. Dawis, L.H. Lofquist, A psychological theory of work adjustment, Minneapolis, MN, University of Minnesota Press, 1984.

[18] M. Durea, R. Strugariu, C. Tammer, On set-valued optimization problems with variable ordering structure, J. Global Optim. 61 (2015), 745-767.

[19] G. Eichfelder, Variable Ordering Structures in Vector Optimization, Springer, Berlin, 2014.

[20] I. Ekeland, On the variational principle, J. Math. Anal. Appl. 47 (1974), 324-353.

[21] C. Gerstewitz (Tammer), Nichtkonvexe Dualität in der Vektoroptimierung, Wiss. Zeitschr. Tech. Hochsch. Leuna-Merseburg 25 (1983), 357-364.

[22] C. Gerth (Tammer), P. Weidner, Nonconvex separation theorems and some applications in vector optimization, J. Optim. Theory Appl. 67 (1990), 297-320.

[23] A. Göpfert, H. Riahi, C. Tammer, C. Zălinescu, Variational methods in partially ordered spaces, SpringerVerlag, New York, 2003.

[24] L.S. Gottfredson, Circumscription and compromise: A developmental theory of occupational aspirations, J. Couns. Psychol. 28 (1981), 545-579.

[25] C. Gutiérrez, G. Kassay, V. Novo, J.L. Ródenas-Pedregosa, Ekeland variational principles in vector equilibrium problems, SIAM J. Optim. 27 (2017), 2405-2425.

[26] P. Hodkinson, A.C. Sparkes, Careership: a sociological theory of career decision making, British J. Sociology Education 18 (1997), 29-44.

[27] M.A. Krasnoselskii, Positive solutions of operator equations. (Translated from the Russian by Richard E. Flaherty; edited by Leo F. Boron), P. Noordhoff Ltd. Groningen, 1964.

[28] J. Jahn, Vector Optimization: Theory, Applications and Extensions, Springer, Heidelberg, 2011.

[29] J. Jahn, T.X.D. Ha, New order relations in set optimization, J. Optim. Theory Appl. 148 (2011), $209-236$.

[30] T.X.D. Ha, Some variants of the Ekeland variational principle for a set-valued map, J. Optim. Theory Appl. 124 (2005), 187-206.

[31] J.C. Kelly, Bitopological spaces, Proc. London Math. Soc. 13 (1963), 71-89.

[32] A. Khan, C. Tammer, C. Zălinescu, Set-Valued Optimization. An Introduction with Applications, Vector Optimization, Springer, Heidelberg, 2015.

[33] K. Klamroth, E. Köbis, A. Schöbel, C. Tammer, A unified approach to uncertain optimization, Euro. J. Oper. Res. 260 (2017), 403-420.

[34] E. Köbis, C. Tammer, Robust vector optimization with a variable domination structure, Carpathian J. Math. 33 (2017), 343-351.

[35] P.Q. Khanh, D.N. Quy, Versions of Ekeland's variational principle involving set perturbations, J. Global Optim. 57 (2013), 951-968.

[36] W.A. Kirk, L.M. Saliga, The Brézis-Browder order principle and extensions of Caristi's theorem, Nonlinear Anal. 47 (2001), 2765-2778.

[37] J.D. Krumboltz, The happenstance learning theory, J. Career Assess. 17 (2009), 135-154.

[38] D. Kuroiwa, On set-valued optimization, Proceedings of the Third World Congress of Nonlinear Analysts, Part 2 (Catania, 2000), Nonlin. Anal. 47 (2001), 1395-1400.

[39] R.W. Lent, S.D. Brown, G. Hackett, Social cognitive career theory, In D. Brown \& Associate (Eds.), Career choice and development (4th ed., pp. 255-311), San Francisco, CA, Jossey-Bass, 2002.

[40] S.A. Leung, The big five career theories, International handbook of career guidance, pp. 115-132, Springer, Dordrecht, 2008. 
[41] D.T. Luc, Theory of Vector Optimization, Springer, Berlin, 1989.

[42] B.S. Mordukhovich, Variational analysis and generalized differentiation, I. Basic Theory, II. Applications, Springer, Berlin, 2006.

[43] B.S. Mordukhovich, Variational Analysis and Applications, Springer, New York, 2018.

[44] M.L. Savickas, Career construction: A developmental theory of vocational behavior, In D. Brown \& Associate (Eds.), Career choice and development (4th ed., pp. 149-205), San Francisco, CA, Jossey-Bass, 2002.

[45] B. Soleimani, C. Tammer, Concepts for approximate solutions of vector optimization problems with variable order structures, Vietnam J. Math. 42 (2014), 543-566.

[46] A. Soubeyran,Variational rationality, a theory of individual stability and change: worthwhile and ambidextry behaviors, Pre-print, GREQAM, Aix Marseille University, 2009.

[47] A. Soubeyran, Variational rationality and the unsatisfied man: routines and the course pursuit between aspirations, capabilities and beliefs, Pre-print, GREQAM, Aix Marseille University, 2010.

[48] A. Soubeyran, Variational rationality. A theory of worthwhile stay and change approach-avoidance transitions ending in traps, Preprint, AMSE, Aix Marseille University, 2016.

[49] A. Soubeyran, Variational rationality.1. An adaptive theory of the unsatisfied man, Preprint, AMSE, Aix Marseille University, 2019.

[50] A. Soubeyran, Variational rationality. 2. A general theory of goals and intentions as satisficing worthwhile moves, Preprint, AMSE, Aix Marseille University, 2019.

[51] D.W. Super, A life-span, life-space approach to career development, J. Vocat. Behav. 16 (1980), 282-298.

[52] A. Pascoletti, P. Serafini, Scalarizing Vector Optimization Problems, J. Optim. Theory Appl. 42 (1984), 499524.

[53] F. Parsons, Choosing a vocation, Boston, Houghton Mifflin, 1909.

[54] J.H. Qiu, On Ha's version of set-valued Ekeland's variational principle, Acta. Math. Sin. 28 (2012), 717-726.

[55] J.H. Qiu, A pre-order principle and set-valued Ekeland variational principle, J. Math. Anal. Appl. 419 (2014), 904-937.

[56] P.L. Yu, Cone convexity, cone extreme points, and nondominated solutions in decision problems with multiobjectives, J. Optim. Theory Appl. 14 (1974), 319-377.

[57] P.L. Yu, Multiple-Criteria Decision Making: Concepts, Techniques and Extensions, Plenum Press, New York, 1985. 